\title{
Modal criteria for optimization of structures through active structural acoustic control
}

\author{
S. Besset and L. Jézéquel \\ LTDS, Équipe D2S, UMR CNRS 5513 \\ École Centrale de Lyon \\ 36 av. Guy de Collongue \\ 69134 Ecully cedex, France
}

International Journal for Numerical Methods in Engineering, 82 (12), 1560-1573, 2010

\begin{abstract}
This paper deals with modal criteria allowing optimization of structures through structural acoustic control (ASAC) based on the independent modal strategy control (IMSC). The aim of the paper is to propose efficient criteria allowing to process optimization of structures by determining the most important modes to control considering only the location of the excitation on the structure. A generalized modal synthesis is used to study the coupled fluid-structure system. Modal criteria are proposed in order to determine the best choice for the controlled modes used in IMSC method. The way the ASAC will be applied is not discussed in the paper. Numerical simulations are computed to show the efficiency of the method, considering a simple vibroacoustic structure.
\end{abstract}

keywords: Modal synthesis; Active control; Vibroacoustic coupling

\section{Introduction}

In order to study vibroacoustic problems without considering too large matrices, many numerical simulation strategies can be found in the literature. Among these techniques, energy methods like SEA or MES are efficient for high frequency range [1, 2, 3. In low and medium frequency domains, modal methods can also be used [4. These modal methods have many advantages. Once modal matrices of the problem have been computed, modal criteria linked to vibroacoustic coupling can easily be defined. The criteria proposed in this paper are derived from those developed by P. Lemerle [5], using Craig \& Bampton's method to describe the dynamic behaviour of a structure. They have already been used to optimize coupled fluid-structure systems in [6, 7].

The modal synthesis technique described here to compute the criteria is based on the "double modal synthesis" proposed by Jézéquel [8, 9] and extended 
to the case of fluid-structure systems [4. This method resorts to a coupling formulation which has been previously investigated [10. An example of modal analysis of a coupled system can be found in [11.

Modal analysis of the structure will lead to modal mass and stiffness matrices, which are then used to obtain effective modal parameters, which in turn lead to criteria allowing the structure to be optimized. The method proposed in this paper may be used for any vibroacoustic system. In fact, the ultimate goal of this approach is to define modal criteria which allow to find the modes that most contribute to the noise level in the cavity. The obtained criteria are related to the coupling terms between the systems different substructures, and are expressed as functions of the terms contained in the modal matrices. Our method is described for the case of a specific system, but can readily be adapted to any vibroacoustic system.

\section{Analysis of the vibroacoustic system}

The structure considered here is a simple structure including plates and an acoustic cavity. This structure is shown on figure 1. The aim of the paper is to optimize the sound level in the cavity by controlling a few modes. The example we give in this section aims at optimizing the pressure level of a few points of the cavity. The method we propose leads to optimization criteria that do not depend on external excitations.

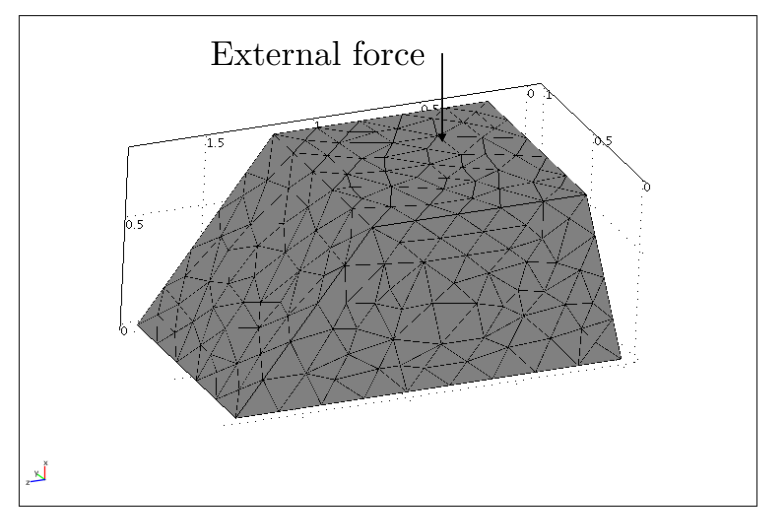

Figure 1: Studied structure 


\begin{tabular}{ccc}
\hline Geometry & Material & Acoustic fluid \\
\hline \hline see figure 1 & $E=2.10^{11} \mathrm{~Pa}$ & air \\
(unit: $\mathrm{m}$ ) & $\nu=0.3$ & $\rho=1.25$ \\
& thickness $=0.01 \mathrm{~m}$ & \\
\hline
\end{tabular}

\subsection{The vibroacoustic problem}

Figure 1 shows the geometry of the vibroacoustic system under consideration. The approach used in this paper to study the vibroacoustic behaviour is a $(\mathbf{u}, p)$ formulation. Suxh kind of formulation can be found in [12. The fluid (air) is considered to be inviscid and leads to a classical linear acoustic problem. The matrix formulation of the problem can be written as follows:

$$
\mathbf{M} \ddot{\xi}+\mathbf{C} \dot{\xi}+\mathbf{K} \xi=\mathbf{A}_{w} \mathbf{w}(t)
$$

$\mathbf{A}_{w} \mathbf{w}(t)$ is the external excitation, located on a plate of the structure. $\mathbf{C}$ is the damping matrix. The aim of the paper is not to deal with the influence of the damping, that is why we consider a very simple viscous modelisation for the structural part of the system. Matrices $\mathbf{M}$ and $\mathbf{K}$ and vector $\xi$ are defined as follows:

$\mathbf{M}=\left[\begin{array}{cc|c}\mathbf{M}_{b b} & \mathbf{M}_{b s} & \mathbf{0} \\ \mathbf{M}_{s b} & \mathbf{M}_{s s} & \\ \hline \mathcal{C}_{F} & \mathbf{M}_{p}\end{array}\right] \quad \mathbf{K}=\left[\begin{array}{cc|c}\mathbf{K}_{b b} & \mathbf{K}_{b s} & -\mathcal{C}_{F}^{T} \\ \mathbf{K}_{s b} & \mathbf{K}_{s s} & \mathbf{K}_{p p}\end{array}\right] \quad \xi=\left\{\begin{array}{c}\mathbf{u}_{b} \\ \mathbf{u}_{s} \\ \mathbf{p}\end{array}\right\}$

where $\mathbf{u}_{b}$ are the degrees of freedom related to the boundaries, $\mathbf{u}_{s}$ are the degrees of freedom related to the surfaces, and $\mathbf{p}$ are the degrees of freedom related to the pressure in the acoustic cavity. Matrices $\mathbf{M}$ and $\mathbf{K}$ defined in equation 2 are mass and stiffness matrices classically used in coupled fluidstructure problems. Degrees of freedom concerning the structure are split into internal degrees of freedom denoted $\mathbf{u}_{s}$ and boundary degrees of freedom denoted $\mathbf{u}_{b}$. This kind of splitting is quite classical in component mode synthesis methods that we will use in the paper.

Equation 1 is a classical interior non-symmetric vibroacoustic equation. The degrees of freedom are displacements for the structural part, and pressure for the acoustic part. The vibroacoustic coupling appears in matrix $\mathcal{C}_{F}$ defined as follows:

$$
\mathcal{C}_{F}=\left[\begin{array}{ll}
\mathbf{M}_{p b} & \mathbf{M}_{p s}
\end{array}\right]=-\left[\begin{array}{l}
\mathbf{K}_{b p} \\
\mathbf{K}_{s p}
\end{array}\right]^{T}
$$

\subsection{Control forces}

The aim of the paper is to define the best criteria for the control law. Thus, a term $\mathbf{e}(t)$ is added in equation 1 . 


$$
\mathbf{M} \ddot{\xi}(t)+\mathbf{C} \dot{\xi}(t)+\mathbf{K} \xi(t)=\mathbf{A}_{w} \mathbf{w}(t)+\mathbf{A}_{e} \mathbf{e}(t)
$$

The control force $\mathbf{A}_{e} \mathbf{e}(t)$ can be provided through many ways (piezoelectric patches for example). Nevertheless, the purpose of the paper is not to propose a method to apply the control forces.

\subsection{Modal synthesis of the system}

The coupled system is studied thanks to component mode synthesis methods that can be found in [13, 4. Although classical component mode synthesis methods are often reliable in the low frequency range, it has been shown in these papers that the component mode synthesis considered here is efficient and allows to deal with medium frequency range. This modal synthesis method aims at modelling the system through cavity modes, plate modes and branch modes. Branch modes are used to describe singularities of the system such as boundaries or stiffeners. As for classical component mode synthesis methods, all the modes are not taken into account and resulting matrices are quite smaller than initial matrices. Thus, generalized degrees of freedom are obtained as follows:

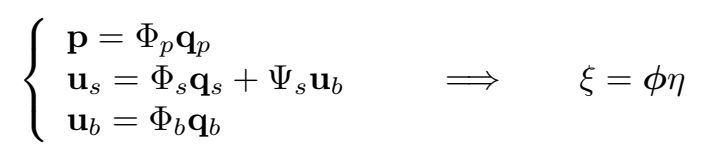

where $\eta=\left\{\begin{array}{l}\mathbf{q}_{b} \\ \mathbf{q}_{s} \\ \mathbf{q}_{p}\end{array}\right\}$ is much smaller than $\xi . \Phi_{s}$ and $\Phi_{p}$ are the matrices of the modes corresponding respectively to the plates and the pressure in the acoustic cavity. $\Psi_{s}$ is the matrix of the static boundary modes. $\Phi_{b}$ is the matrix of the "branch modes" of the structure as defined in [4].

In fact, three modal synthesis are successively processed. First, considering acoustic modes $\Phi_{p}$, a transfer matrix $\mathbf{T}_{1}$ is defined as follows:

$$
\mathbf{T}_{1}=\left[\begin{array}{ccc}
\mathbf{I d} & 0 & 0 \\
0 & \mathbf{I d} & 0 \\
0 & 0 & \Phi_{p}
\end{array}\right]
$$

where Id is the identity matrix. Thus, mass and stiffness matrices can be expressed as follows:

$$
\mathbf{M}_{1}=\mathbf{T}_{1}^{T} \mathbf{M} \mathbf{T}_{1} \quad \text { and } \quad \mathbf{K}_{1}=\mathbf{T}_{1}^{T} \mathbf{K} \mathbf{T}_{1}
$$

Matrices $\mathbf{M}_{1}$ and $\mathbf{K}_{1}$ can be split as follows:

$$
\mathbf{M}_{1}=\left[\begin{array}{lll}
\mathbf{M}_{1 b b} & \mathbf{M}_{1 b s} & 0 \\
\mathbf{M}_{1 s b} & \mathbf{M}_{1 s s} & 0 \\
\mathbf{M}_{1 p b} & \mathbf{M}_{1 p s} & \mathbf{M}_{1 p p}
\end{array}\right] \quad \text { and } \quad \mathbf{K}_{1}=\left[\begin{array}{ccc}
\mathbf{K}_{1 b b} & \mathbf{K}_{1 b s} & \mathbf{K}_{1 b p} \\
\mathbf{M}_{1 s b} & \mathbf{M}_{1 s s} & \mathbf{K}_{1 s p} \\
0 & 0 & \mathbf{K}_{1 p p}
\end{array}\right]
$$


A second modal synthesis is then processed considering transfer matrix $\mathbf{T}_{2}$ :

$$
\mathbf{T}_{2}=\left[\begin{array}{ccc}
\mathbf{I d} & 0 & 0 \\
\Psi_{s} & \Phi_{s} & 0 \\
0 & 0 & \mathbf{I d}
\end{array}\right]
$$

where $\Psi_{s}=\mathbf{K}_{1 s s}{ }^{-1} \mathbf{K}_{1 s b}$ (Craig \& Bampton theory) and $\Phi_{s}$ are the modes of the structure given by the following equation:

$$
\left(\mathbf{K}_{1 s s}-\left[\omega^{2}\right] \mathbf{M}_{1 s s}\right) \Phi_{s}=0
$$

The last modal synthesis aims at expressing $\mathbf{u}_{b}$ in function of branch modes $\Phi_{b}$ as explained in [13, 4.

Thanks to relations 5 we can write the modal equation of motion as follows:

$$
\overline{\mathbf{M}} \ddot{\eta}+\overline{\mathbf{C}} \dot{\eta}+\overline{\mathbf{K}} \eta=\overline{\mathbf{A}}_{w} \mathbf{w}(t)+\overline{\mathbf{A}}_{e} \mathbf{e}(t)
$$

where:

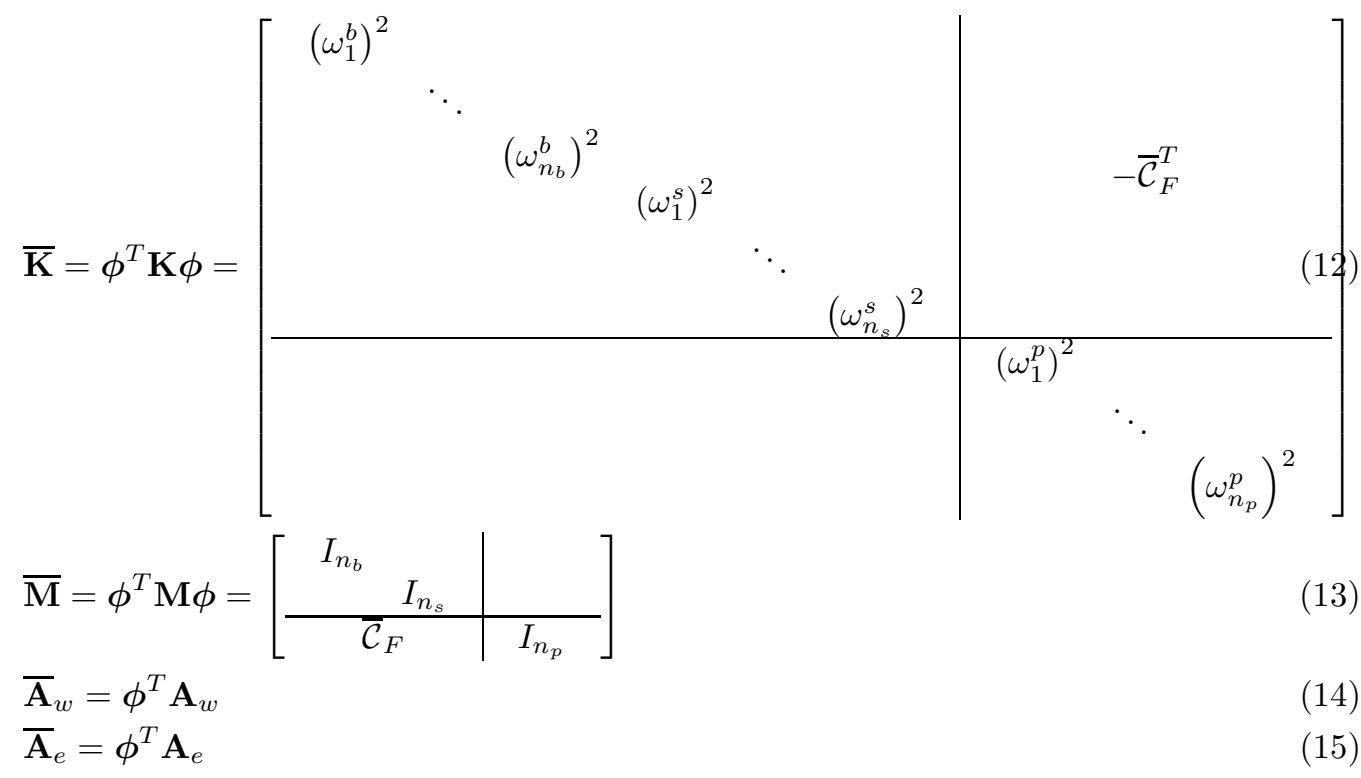

Matrix $\overline{\mathbf{C}}=\boldsymbol{\phi}^{T} \mathbf{C} \boldsymbol{\phi}$ is considered to be diagonal thanks to Basile's hypothesis. Equation 11 can be rewritten using vector $X=\left\{\begin{array}{l}\eta \\ \dot{\eta}\end{array}\right\}$ :

$$
\dot{X}=\mathbf{D} X+\mathbf{E w}(t)+\mathbf{F e}(t)
$$

where $\mathbf{D}, \mathbf{E}$ and $\mathbf{F}$ are defined as follows:

$$
\mathbf{D}=\left[\begin{array}{cc}
\mathbf{0} & \overline{\mathbf{M}} \\
-\overline{\mathbf{K}} & -\overline{\mathbf{C}}
\end{array}\right], \quad \mathbf{E}=\left[\begin{array}{c}
\mathbf{0} \\
\overline{\mathbf{A}}_{e}
\end{array}\right] \quad \text { and } \quad \mathbf{F}=\left[\begin{array}{c}
\mathbf{0} \\
\overline{\mathbf{A}}_{w}
\end{array}\right]
$$




\section{$3 \quad$ Vibroacoustic criteria}

The aim of the method proposed in this paper is to use vibroacoustic criteria to determine which modes should be controlled. Thus, let define these criteria from equation [1]. As there is no noise source inside the cavity, the $\mathrm{n}^{\mathrm{e}}$ line of equation 11 leads to:

$$
-\omega^{2} \overline{\mathbf{M}}_{p b}^{n} \mathbf{q}_{b}-\omega^{2} \overline{\mathbf{M}}_{p s}^{n} \mathbf{q}_{s}+\left(\left(\omega_{p}^{n}\right)^{2}-\omega^{2}+i \omega \bar{c}_{n}\right) \mathbf{q}_{p}^{n}=0
$$

The assumption "there is no noise source in the cavity" is not only made for the sake of brevity and clarity. The problem we consider in this paper deals with the noise in acoustic cavities and should be applied to aircrafts or automotive industry. Thus, the only excitation sources we consider are external excitations on the boundaries of the structure.

The noise level is linked to the pressure in the acoustic cavity. The pressure can be expressed as follows:

$$
\mathbf{p}=\sum_{n=1}^{N_{p}} \Phi_{p}^{n} \mathbf{q}_{p}^{n}
$$

where $N_{p}$ is the number of acoustic modes computed in the modal synthesis. Substituting equation 18 in equation 19, we obtain the following relation linking the pressure in the cavity with the generalized degrees of freedom $\mathbf{q}_{b}$ and $\mathbf{q}_{s}$ :

$$
\mathbf{p}=\sum_{n=1}^{N_{p}} \frac{\omega^{2} \Phi_{p}^{n}\left(\overline{\mathbf{M}}_{p b}^{n} \mathbf{q}_{b}+\overline{\mathbf{M}}_{p s}^{n} \mathbf{q}_{s}\right)}{\left(\omega_{p}^{n}\right)^{2}-\omega^{2}+i \omega \bar{c}_{n}}
$$

The aim is to express the pressure $\mathbf{p}$ in function of degrees of freedom $\mathbf{u}_{b}$ and $\mathbf{u}_{s}$ in order to define vibroacoustic paths as defined in [7. These various "vibration paths" are linked to the different ways of propagation of the vibration that cause the noise into the cavity. $\mathbf{q}_{b}$ and $\mathbf{q}_{s}$ can then be expressed thanks to relation 5

$$
\left\{\begin{array}{l}
\mathbf{q}_{b}=\tilde{\Phi}_{b} \mathbf{u}_{b} \\
\mathbf{q}_{s}=\tilde{\Phi}_{s}\left(\mathbf{u}_{s}-\Psi_{s} \mathbf{u}_{b}\right)
\end{array}\right.
$$

where $\tilde{\Phi}_{b}$ and $\tilde{\Phi}_{s}$ are pseudo-inverse of matrices $\Phi_{b}$ and $\Phi_{s}$. Thus, two criteria can be defined, corresponding to the relation between the pressure and the degrees of freedom related to the surfaces and the boundaries:

$$
\begin{aligned}
C_{b} & =\sum_{n=1}^{N_{p}}\left\|\frac{\omega_{p}^{n} \Phi_{p}^{n}\left(\overline{\mathbf{M}}_{p b}^{n} \tilde{\Phi}_{b}-\overline{\mathbf{M}}_{p s}^{n} \tilde{\Phi}_{s} \Psi_{s}\right)}{\bar{c}_{n}}\right\| \\
C_{s} & =\sum_{n=1}^{N_{p}}\left\|\frac{\omega_{p}^{n} \Phi_{p}^{n} \overline{\mathbf{M}}_{p s}^{n} \tilde{\Phi}_{s}}{\bar{c}_{n}}\right\|
\end{aligned}
$$


In the following, we will only consider criterion $C_{s}$ for the control forces that will be applied on the structure. This criterion is linking the pressure $\mathbf{p}$ and the degrees of freedom of the structure $\mathbf{u}_{s}$. Thus, this criterion is not directly linked to the applied excitation forces appearing in equation 4. This should be a problem since the control we consider in the paper is applied through forces. Nevertheless, the control force $\mathbf{f}=\mathbf{A}_{e} \mathbf{e}(t)$ can be linked to the displacement of the structure $\mathbf{u}_{s}$ through the following equation:

$$
\mathbf{f}=\mathbf{H}(\omega) \mathbf{u}_{s} \Rightarrow \mathbf{u}_{s}=\tilde{\mathbf{H}}(\omega) \mathbf{f}
$$

where $\mathbf{H}(\omega)$ corresponds to the dynamic response of the structure. Thus, the link between $\mathbf{f}$ and $\mathbf{u}_{s}$ should be expressed through criterion $\tilde{C}_{s}$ :

$$
\tilde{C}_{s}=\sum_{n=1}^{N_{p}}\left\|\frac{\omega_{p}^{n} \Phi_{p}^{n} \overline{\mathbf{M}}_{p s}^{n} \tilde{\Phi}_{s} \tilde{\mathbf{H}}(\omega)}{\bar{c}_{n}}\right\|=\|\tilde{\mathbf{H}}(\omega)\| C_{s}
$$

In the following, we consider that considering criterion $C_{s}$ is enough to define the right control law.

Criterion $C_{s}$ can be split into $N_{s}$ criteria denoted $C_{s}^{j}$ corresponding to the $N_{s}$ modes of the structure:

$$
C_{s}^{j}=\sum_{n=1}^{N_{p}}\left\|\frac{\omega_{p}^{n} \Phi_{p}^{n} \overline{\mathbf{M}}_{p s}^{n} \tilde{\Phi}_{s}^{j}}{\bar{c}_{n}}\right\| \quad 1 \leq j \leq N_{s}
$$

where $\tilde{\Phi}_{s}^{j}$ is the $j^{\mathrm{e}}$ column of matrix $\tilde{\Phi}_{s}$. Considering these $N_{s}$ criteria, it is possible to evaluate the most important modes of the structure for the noise level. Figure 2 shows the values of the criteria for $N_{s}=40$.

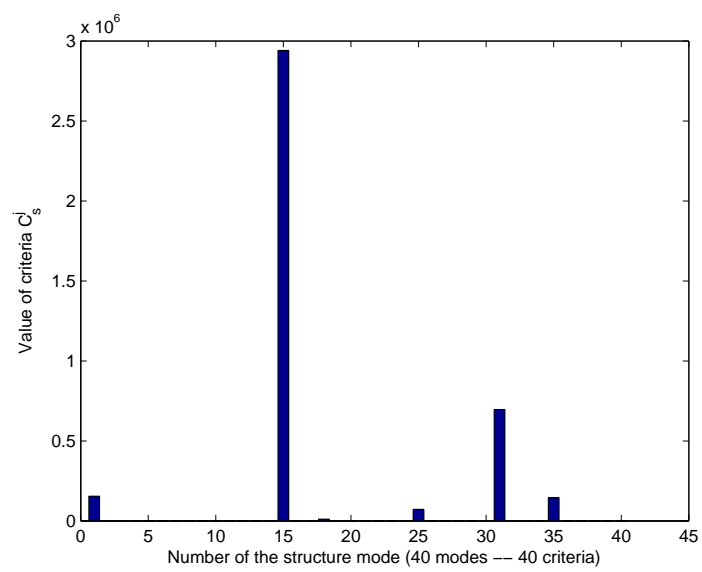

Figure 2: Values of criteria $C_{s}^{j}$ 


\section{Active control of the structure}

\subsection{Method used for the control}

The aim of the paper is to use the modal criteria defined in section 3 to control the modes of the structure. The active control method we consider here can be found in [14, 15, 16.

We recall that the proposed method allows to define criteria that do not depend on the excitation. Thus, we don't expect better results than other control methods for the results would depend of the considered excitation, but we expect noise levels to be reduced in order to validate the criteria.

In order to be able to control each mode separately, it is useful to define a matrix $\mathbf{L}_{e}$ so that:

$$
\overline{\mathbf{A}}_{e} \mathbf{L}_{e}=I \quad \Longrightarrow \quad \mathbf{e}(t)=\mathbf{L}_{e} \mathbf{r}(t)
$$

Equation 16 can then be rewritten as follows:

$$
\dot{X}=\mathbf{D} X+\mathbf{E w}(t)+\left[\begin{array}{l}
\mathbf{0} \\
I
\end{array}\right] \mathbf{r}(t)
$$

where $I$ is the identity matrix. The dimension of vector $\mathbf{r}(t)$ corresponds to the number of control forces. The control excitation is then expressed in function of the generalized degrees of freedom $\eta$ and $\dot{\eta}$ :

$$
\mathbf{r}(t)=-\mathbf{G} \eta-\mathbf{H} \dot{\eta}
$$

$\mathbf{G}$ and $\mathbf{H}$ are diagonal matrices that have to be found so that matrix $\left[\begin{array}{c}\mathbf{0} \\ I\end{array}\right]$ can be expressed as follows:

$$
\left[\begin{array}{l}
\mathbf{0} \\
I
\end{array}\right] \mathbf{r}(t)=\left[\begin{array}{cc}
\mathbf{0} & \mathbf{0} \\
-\mathbf{G} & -\mathbf{H}
\end{array}\right]\left\{\begin{array}{l}
\eta \\
\dot{\eta}
\end{array}\right\}
$$

In order to find values for matrices $\mathbf{G}$ and $\mathbf{H}$, we will consider $m$ criteria $J_{m}$ to control the structure, corresponding to the $m$ modes to attenuate. These modes have been found using the vibroacoustic criteria defined in section 3 . Criteria $J_{m}$ can be expressed as follows:

$$
J_{m}=\lim _{t \rightarrow \infty} E\left(\frac{1}{t} \int_{0}^{t}\left[\begin{array}{c}
\eta_{m} \\
\dot{\eta}_{m} \\
r_{m}
\end{array}\right]^{T}\left[\begin{array}{ccc}
\omega_{m}^{2} & 0 & 0 \\
0 & I & 0 \\
0 & 0 & R_{m}
\end{array}\right]\left[\begin{array}{l}
\eta_{m} \\
\dot{\eta}_{m} \\
r_{m}
\end{array}\right] d t\right)
$$

where $R_{m}$ is a value that is arbitrarily chosen (the efficiency of the control will depend on this value). This relation leads to the following expression of $r_{m}(t)$, that can be found in [16]: 


$$
\begin{aligned}
& r_{m}(t)=\omega_{m}\left[\omega_{m}-\left(\omega_{m}^{2}+R_{m}^{-1}\right)^{\frac{1}{2}}\right] \eta_{m}(t) \\
& +\left(c_{m} \omega_{m}-\left[c_{m}^{2} \omega_{m}^{2}+\left(2 \omega_{m}\left[-\omega_{m}+\left(\omega_{m}^{2}+R_{m}^{-1}\right)^{\frac{1}{2}}\right]\right)+R_{m}^{-1}\right]^{\frac{1}{2}}\right) \dot{\eta}_{m}(t)
\end{aligned}
$$

Which allows to find values for matrices $\mathbf{G}$ and $\mathbf{H}$.

\subsection{Results}

Considering figure 2, we chose to attenuate four modes of the structure. The results of the control are given on figures 3 and 4 . The structure considered was presented in figure 1. A force have been applied on this structure (see figure 1), and we computed the frequency response (see figure 3) and the temporal response (see figure 4) to obtain the pressure in the cavity. The calculations have been made considering the average pressure in a small volume in the cavity.

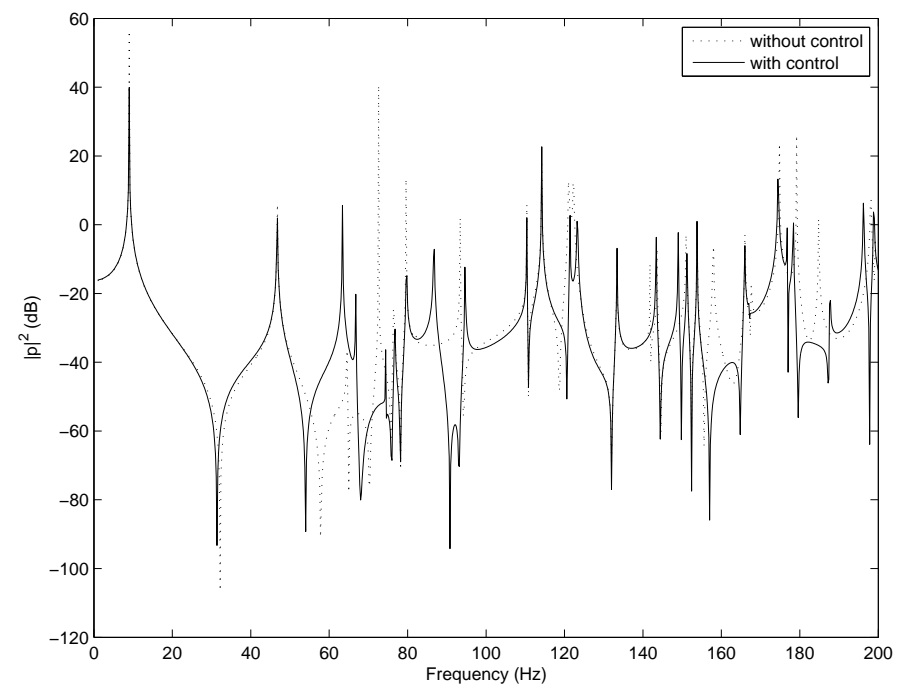

Figure 3: Square pressure in the acoustic cavity - FRF

Figure 4 shows the noise level, that is linked to $|p|^{2}$, is quite attenuated with the active control, even if only four modes are controlled. Moreover, another calculation has been made, considering 20 modes to be attenuated (see figures 5 and 6). This seems to be too much for the control to be efficient. The frequency response function (see figure 5) shows that the control is effective around 170 $\mathrm{Hz}$, but this is not the case in lower frequency range, for example around $65 \mathrm{~Hz}$. Moreover, figure [6] shows the result with control is worth than without control. This effect can be explained by the fact that the control law has to deal with 

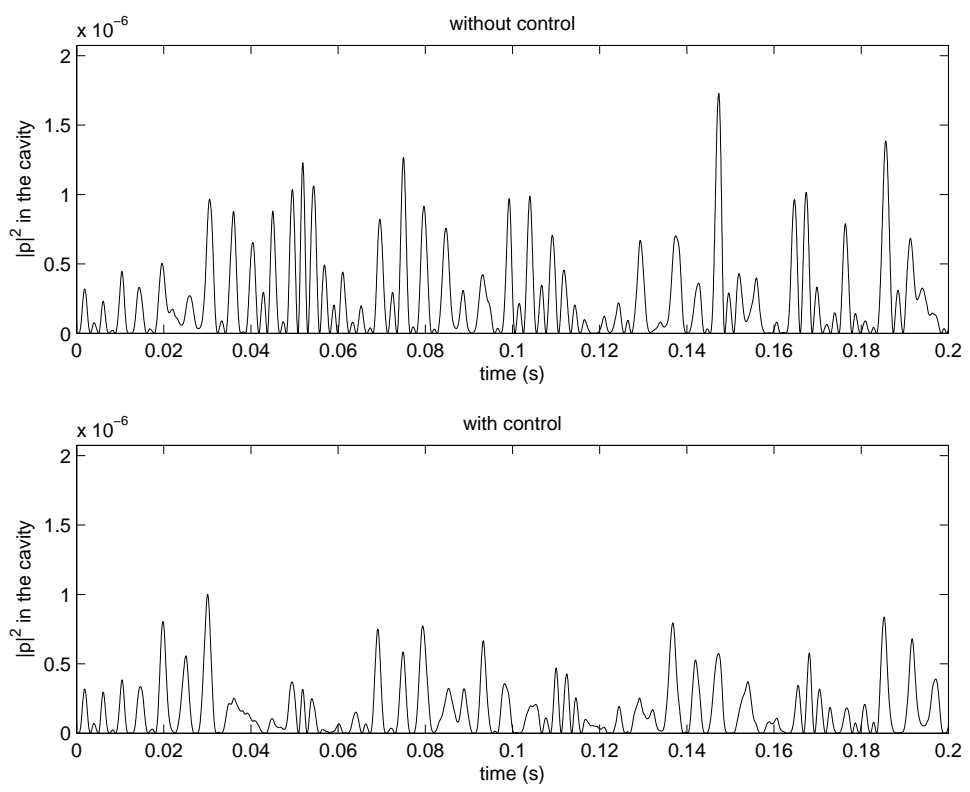

Figure 4: Square pressure in the acoustic cavity -4 modes controlled

modes that do not affect the behaviour of the structure and that should not be controlled.

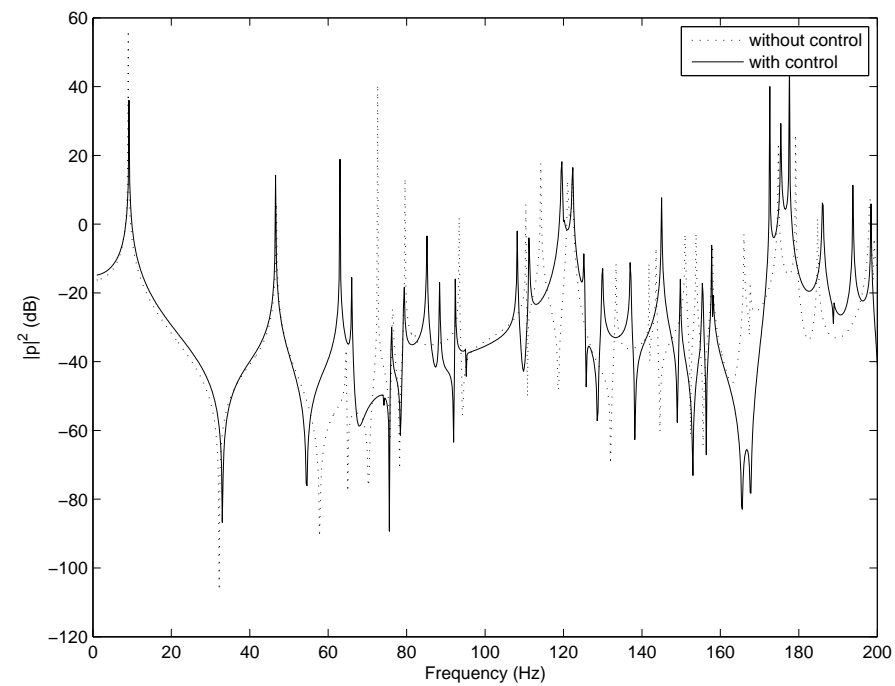

Figure 5: Square pressure in the acoustic cavity - FRF - 20 modes controlled 

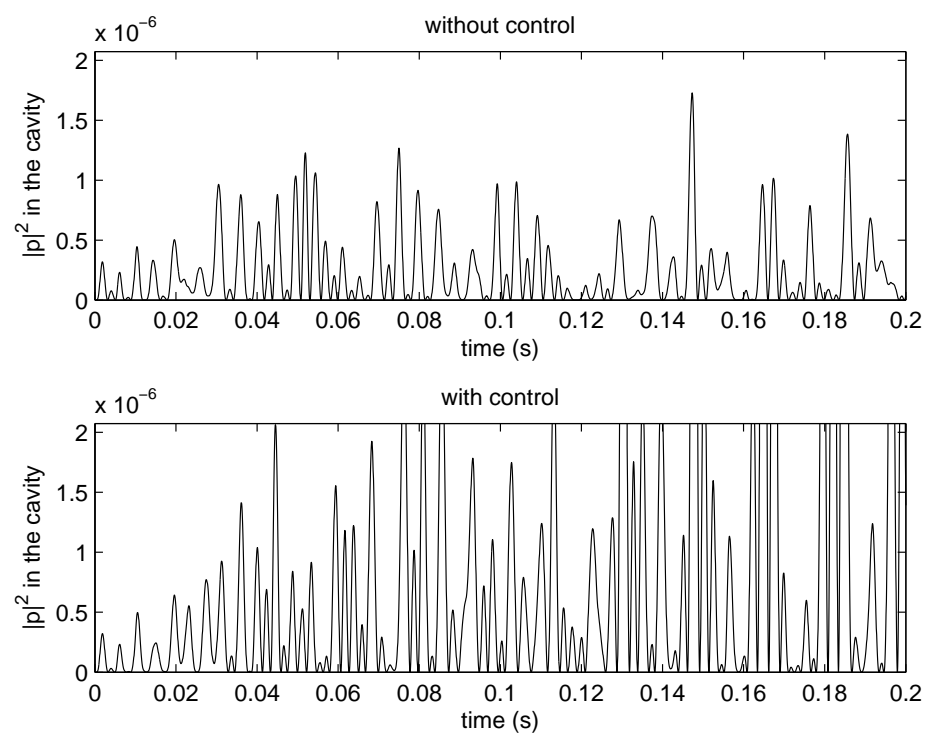

Figure 6: Square pressure in the acoustic cavity - 20 modes controlled

It is also possible to see the influence of the control of each of the 4 controlled modes. Figures 7 and 8 show the frequency response functions of the system for each case. Figures 9 and 10 show the efficiency of the control. Controlling these modes allows the pressure level in the cavity to decrease: criterion $C_{s}$ is efficient to detect the modes to control.

It is interesting to observe that although the value of the criteria shown in figure 2 for the 15 th mode is much higher than for the 25 th mode. However, it seems contradictory withe the results shown in figures 9 and 10. In fact, figure 7 shows that controlling the 15th mode leads to higher pressure levels around 65 $\mathrm{Hz}$, which explains that the control is less efficient that for the 25 th mode.

\section{Conclusion}

Vibroacoustic criteria have been defined and used in the case of active control of a structure. We show that these criteria allow to find the most important modes in fluid-structure coupling. Thus, treating only the four main modes, it is possible to significantly reduce the noise level in a cavity.

Moreover, we show that considering all the modes does not produce better results. Finding the most important modes is thus very important. The control should be processed thanks to piezoelectric patches for example, which could be made in future works.

At last, the results presented in the paper are related to a single example. It 


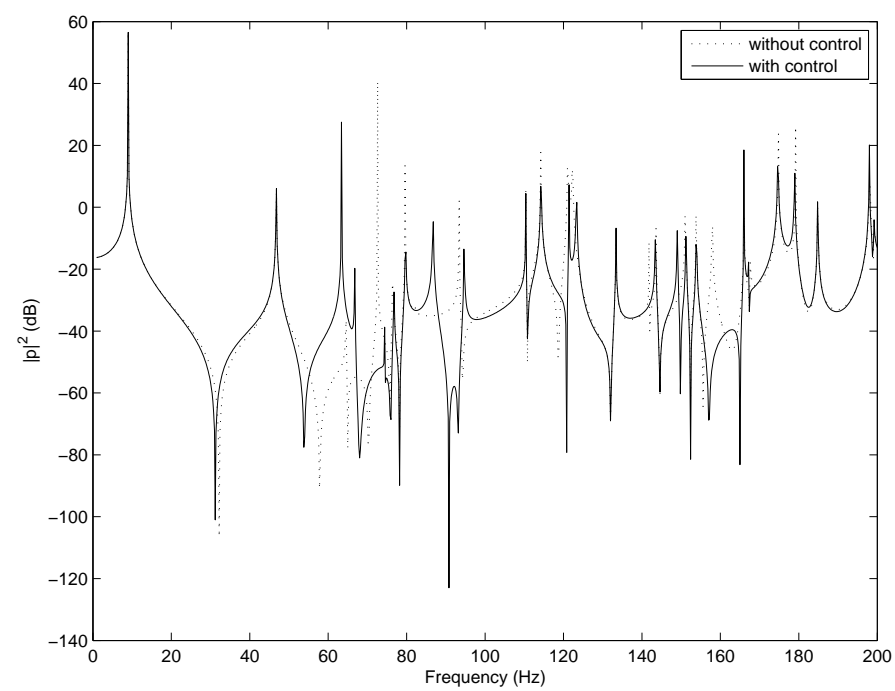

Figure 7: Square pressure in the acoustic cavity $-\mathrm{FRF}-15^{\text {th }}$ mode controlled

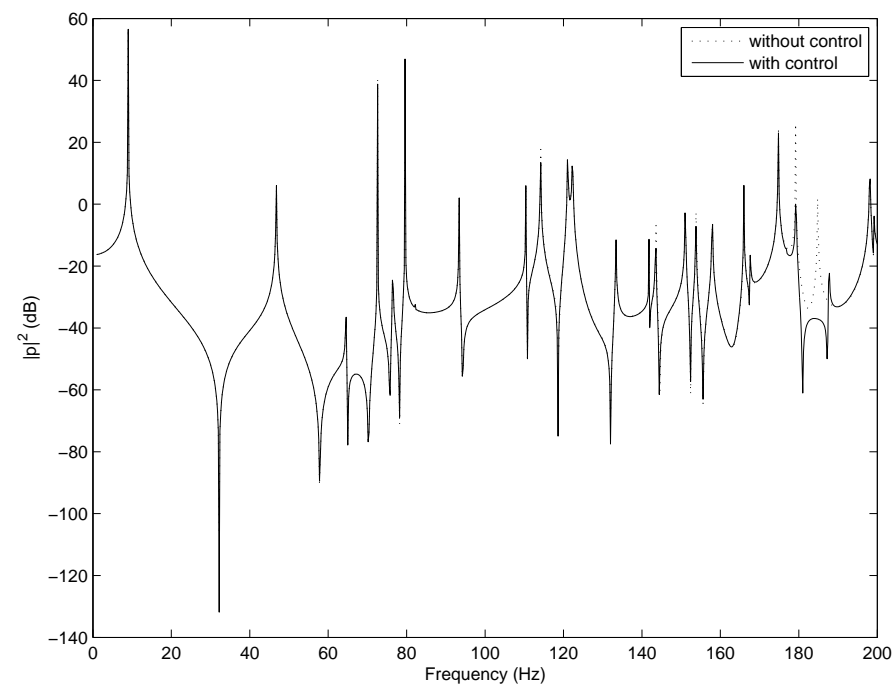

Figure 8: Square pressure in the acoustic cavity $-\mathrm{FRF}-25^{\text {th }}$ mode controlled

would be quite interesting to study in what extent these results are influenced by the type and the location of the excitation. 

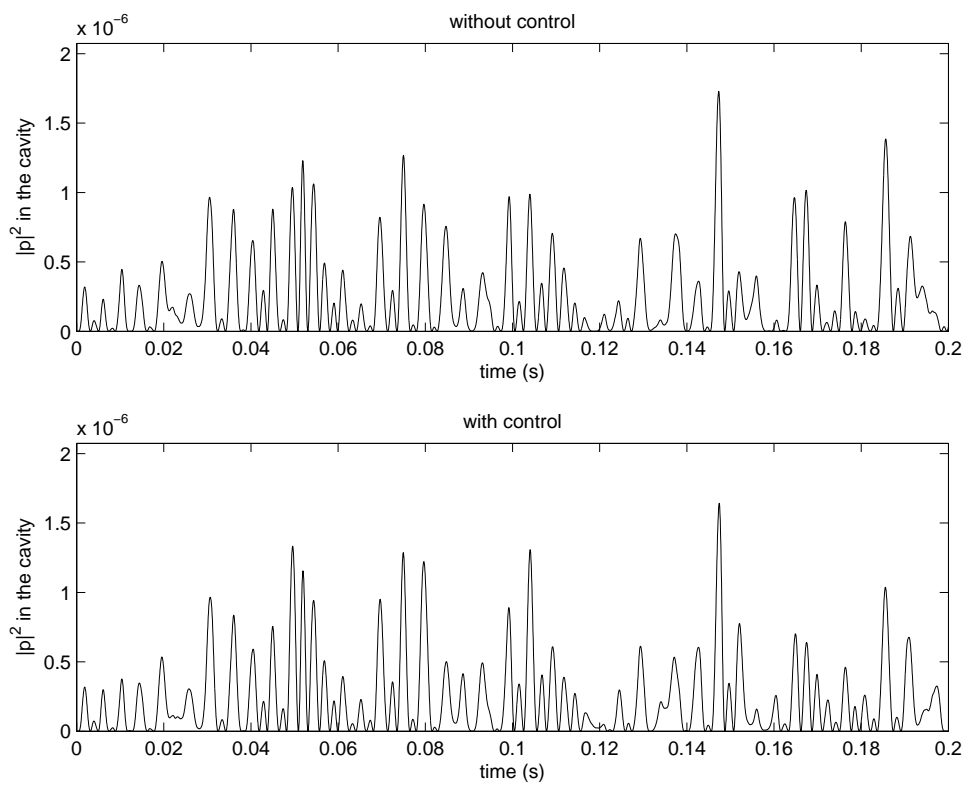

Figure 9: Square pressure in the acoustic cavity $-15^{\text {th }}$ mode controlled

\section{References}

[1] R. H. Lyon, Statistical Energy Analysis of Dynamical Systems: Theory and Application, MIT Press, Cambridge, Massachussetts, 1975.

[2] A. Wang, N. Vlahopoulos, K. Wu, Development of an energy boundary element formulation for computing high-frequency sound radiation from incoherent intensity boundary conditions, Journal of Sound and Vibration 278 (1-2) (2004) 413-436.

[3] V. Cotoni, A. L. Bot, L. Jézéquel, High frequency radiation of 1-shaped plates by a local energy flow approach, Journal of Sound and Vibration 250 (3) (2002) 431-444.

[4] S. Besset, L. Jézéquel, Vibroacoustical analysis based on a multimodal strategy: Triple modal synthesis, J. Vib. Acoust 130 (3).

[5] P. Lemerle, H. Grangier, L. Jézéquel, Optimization of structures to reduce the transmission of vibrations : on approach based on kuhn-tucker optimality conditions with constraints on modal effective parameters, in: 9th International Modal Analysis Conference, 1991.

[6] S. Besset, L. Jézéquel, Optimization of structural dynamic behaviour based on effective modal parameters, International Journal for Numerical Methods in Engineering 70 (5) (2007) 523-542. 

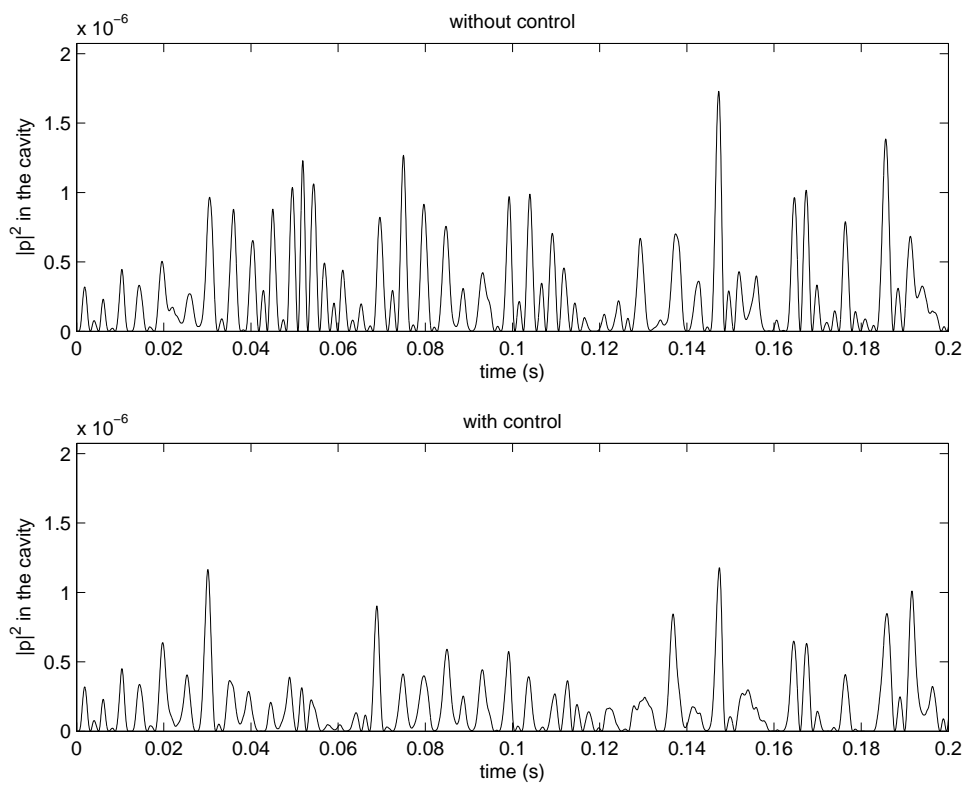

Figure 10: Square pressure in the acoustic cavity $-25^{\text {th }}$ mode controlled

[7] S. Besset, L. Jézéquel, Modal criteria for optimization of the acoustical behaviour of a vibroacoustic system based on a systemic approach, International Journal for Numerical Methods in Engineering 73 (2008) 1347-1373.

[8] L. Jézéquel, H. D. Setio, Component modal synthesis methods based on hybrid models, part i : Theory of hybrid models and modal truncation methods, ASME Journal of Applied Mechanics 61 (1994) 100-108.

[9] L. Jézéquel, H. D. Setio, Component modal synthesis methods based on hybrid models, part ii : Numerical tests and experimental identification of hybrid model, ASME Journal of Applied Mechanics 61 (1994) 109-116.

[10] R. Ohayon, Reduced models for fluid-structure interaction problems, International Journal for Numerical Methods in Engineering 60 (2003) 139-152.

[11] G. E. Sandberg, P.-A. Hansson, M. Gustavsson, Domain decomposition in acoustic and structure-acoustic analysis, Computer Methods in Applied Mechanics and Engineering 190 (2001) 2979-2988.

[12] H. J. Morand, R. Ohayon, Fluid Structure Interaction, Wiley \& Sons, 1995.

[13] S. Besset, L. Jézéquel, Dynamic substructuring based on a double modal analysis, J. Vib. Acoust 130 (1). 
[14] L. Jézéquel, V. Roberti, Optimal preview semiactive suspension, Journal of dynamic systems, measurements and control 118 (1) (1996) 99-105.

[15] V. Roberti, L. Jézéquel, Adaptive semiactive isolation of seismic buildings, Smart Materials and Structures 4 (1A) (1995) A32-A40.

[16] M. Collet, L. Jézéquel, J. Perisse, Independant modal space control of a thin plate with piezoelectrics as sensors and actuators, in: IMAC XIII, Nashville, Tennessee, 1995. 